\title{
1 Traffic simulation for all: a real world traffic scenario from the city of Bologna
}

Laura Bieker, Daniel Krajzewicz, AntonioPio Morra, Carlo Michelacci and Fabio

Cartolano;German Aerospace Center, Rutherfordstraße 2, 12489 Berlin, Germany; Comune

di Bologna, Bologna, Italy

\{Laura.Bieker, Daniel.Krajzewic\}@DLR.de;

\{AntonioPio.Morra, Carlo.Michelacci\}@comune.bologna.it; cartolano@ibinnovation.eu;

\subsection{Abstract}

It is time consuming to set up a realistic traffic simulation scenario. Even though data is available, a large effort is needed to gather, convert and adapt all the data needed to replicate a part of a real road network. Available road networks usually have to be corrected and adapted to the used simulation model. The demand has to be converted or even generated using given measurements. The measurements must be imported into the simulation system's architecture to allow the models' calibration and validation. Additional road side structures must be converted into a proper representation and embedded into the scenario. Therefore, three real world traffic simulation scenarios of the city of Bologna are made available to the public. With these scenarios researchers are able to start their investigations with little preparation effort and can concentrate on their research questions.

Keywords: Real world traffic scenario, open data, validation

\subsection{Traffic simulation and Open Data}

For modelling real world scenarios the traffic simulation needs input traffic and infrastructure data about the real-world traffic conditions. The quality of the input data is crucial for realistic simulation results. Therefore the following input data for the simulation are needed:

1. Representation of the road network

2. Representation of the demand

3. Representation of real traffic lights

4. Representation of infrastructure

Without these representations a realistic traffic simulation is hardly possible. But collecting, processing and validating the input data is time consuming. Sometimes it is difficult to receive real world data especially the real traffic light signal plans are rarely open to the public. Consequently, it is difficult for traffic researchers to analyse and evaluate their traffic light algorithm under real world conditions. Thus, real world scenarios from Bologna are prepared and described in this work and will be made publicly available within the SUMO package [1] [2]. By making the scenarios available to the public the scenarios can be used for further research with little effort. 


\subsection{Bologna Scenarios}

The simulation scenarios presented in this paper have been built in the project iTETRIS ("An Integrated Wireless and Traffic Platform for Real-Time Road Traffic Management Solutions"). TTETRIS was co-funded by the European Commission between 2008 and 2011 and was concerned in developing a simulation system for evaluations of large-scale traffic management solutions that work via vehicular communications [3]. A large part of the project was dedicated to determining and modelling of real-world traffic. Major contribution on this task was performed by the municipality of Bologna who was a project partner in iTETRIS. Besides describing the situation and the problems in Bologna, this municipality also delivered initial ideas for traffic management applications and additionally a large set of data and simulation scenarios.

The given data included representations of the areas around the "Andrea Costa" and the "Pasubio" roads, as input files for the commercial microscopic traffic simulation Vissim, a product of PTV AG. Each of the scenarios included the demand for Bologna's peak hour (8:00am - 9:00am). Additional data sets supported by the municipality of Bologna included positions of traffic lights, traffic light plans, inductive loop positions and measures and many others. A further scenario, "joined", was implemented within iTETRIS by merging both Vissim scenarios.

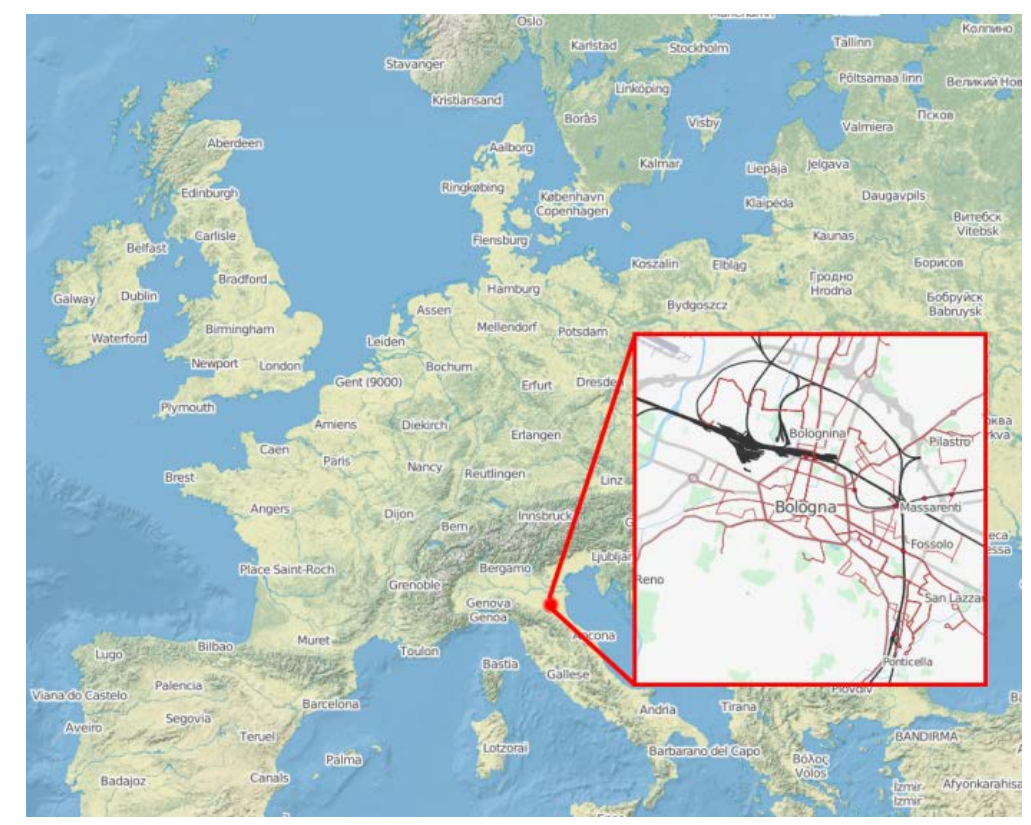

Figure 2-1: Location of Bologna

In the following the three regions of Bologna and the traffic simulation scenario are described.

\subsubsection{Andrea Costa}

The Andrea Costa scenario includes the area around the football stadium and was set up to simulate the mobility of big events such as football matches or concerts. 
Table 2-1: Selected views on the A. Costa scenario from iTETRIS

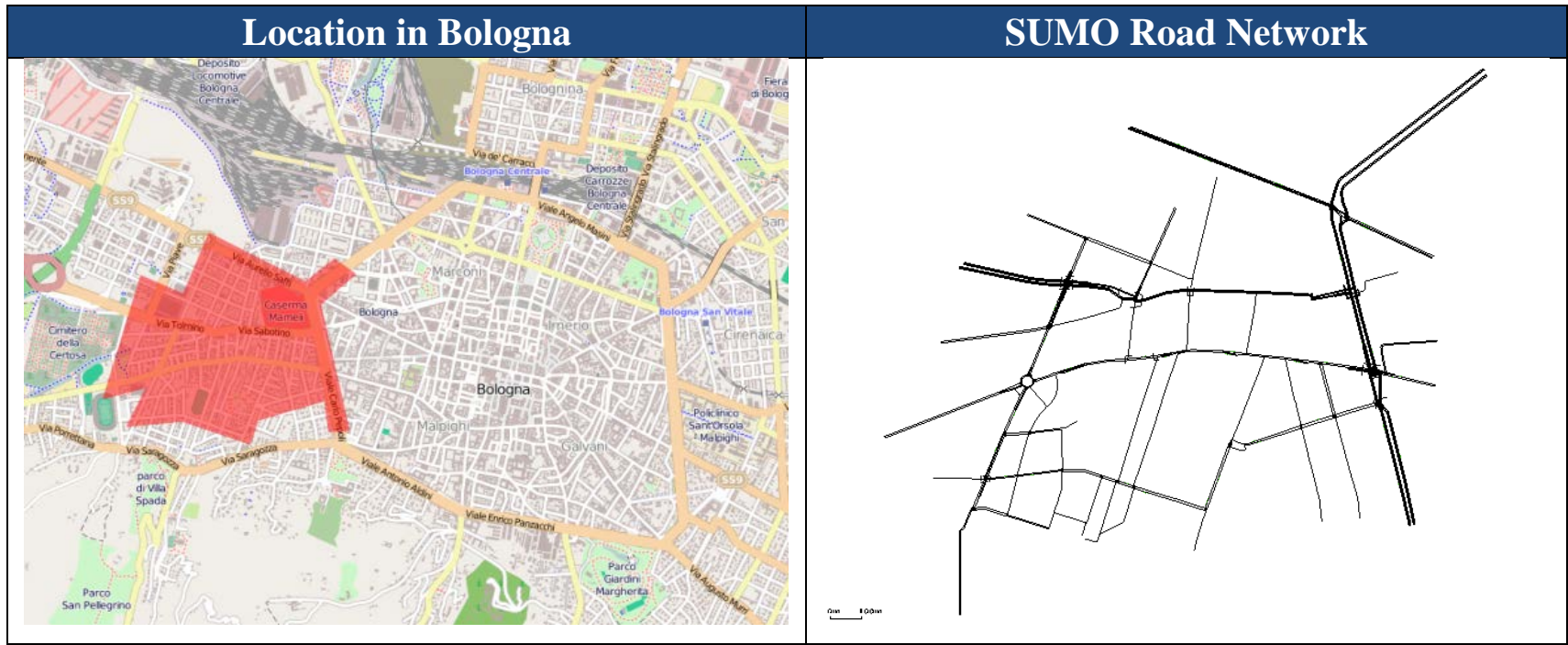

\subsubsection{Pasubio}

The Pasubio scenario extends the Andrea Costa scenario about the area around the hospital and includes also common routes to the football stadium.

Table 2-2: Selected views on the Pasubio scenario from iTETRIS

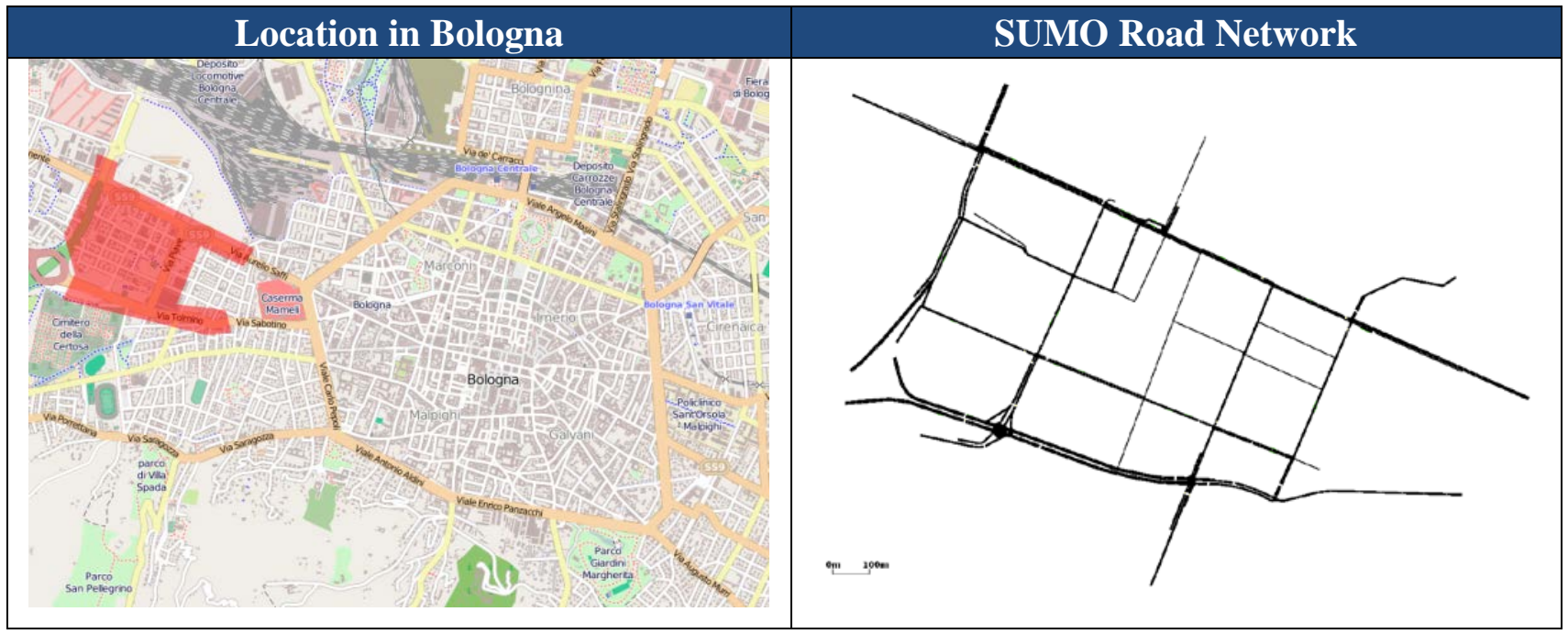

\subsubsection{Andrea Costa and Pasubio joined scenario}

Because of the relatively small areal size of the VISSIM networks "Andrea Costa" and "Pasubio", it was decided to generate one scenario based on both. This scenario should be obtained by joining both given, as the areas they cover are overlapping. In addition to the traffic demand for the morning traffic demand, the Andrea Costa-Pasubio joined scenario also includes the traffic demand for a football match. For generating a higher traffic demand the induction loop data from 24 March 2010 when a football match took place was compared to the traffic flow on the day one week in prior. Additional routes were generated from the analysed data set. 
Table 2-3: Selected views on the "joined" scenario from iTETRIS

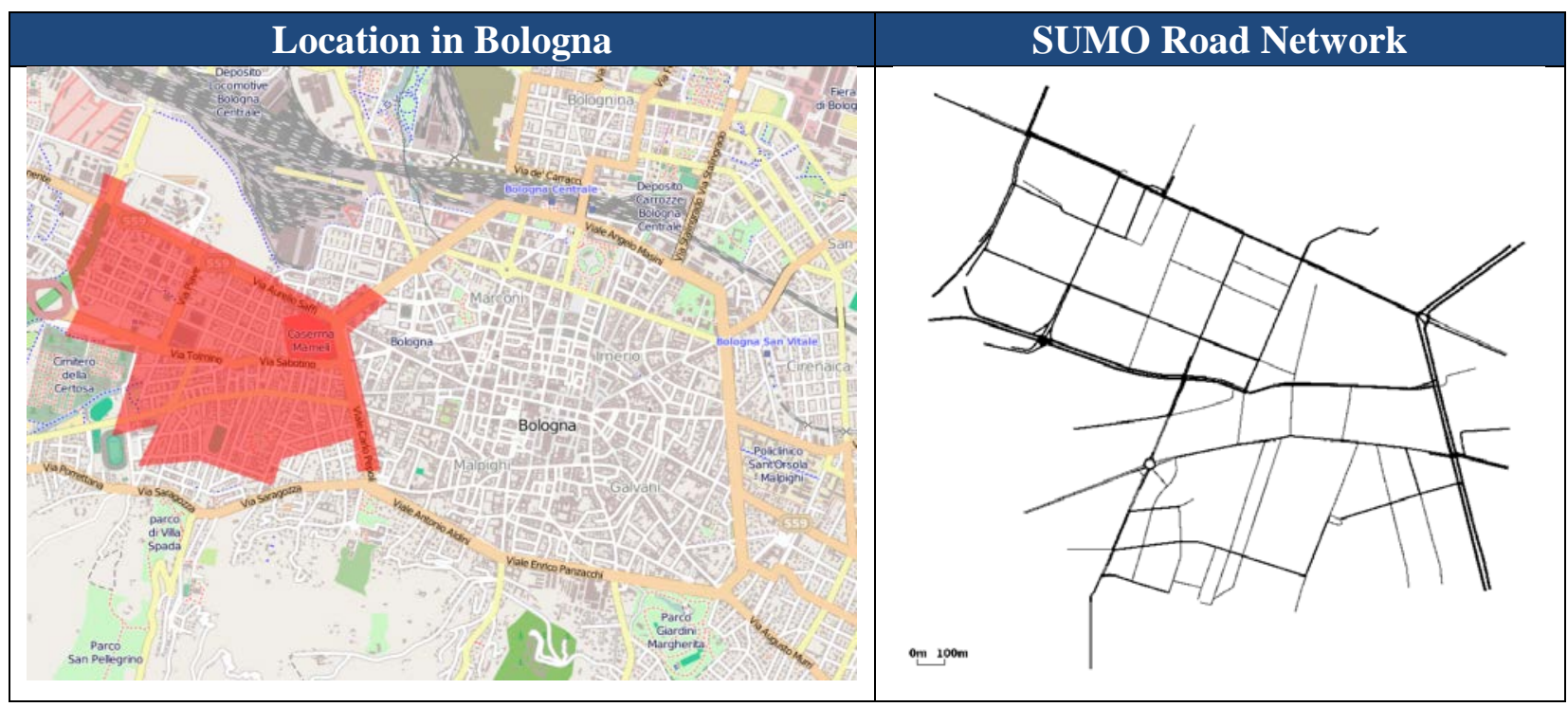

\subsection{Development of the Scenario}

\subsubsection{Traffic Network}

The supported VISSIM scenarios model two areas located east to the inner city ring. They describe a slightly pruned road network not containing few smaller streets. Traffic lights are defined, including their positions and signal plans. Passenger vehicles in each network are described in an aggregated manner: the numbers of vehicles to insert are given for certain roads located at the network's border. Following their initial route, the vehicles pass certain "routing decision points" at which they get a new route assigned randomly, according to a given distribution. This method is used for reproducing the turn percentages at intersections measured in reality. Both scenarios describe the traffic at the morning peak hour, between 8:00 a.m. and 9:00 a.m.

In addition to the passenger vehicles, both scenarios include a description of the public bus transport taking place in the regarded area. Both, positions of the bus stops and bus routes and schedules are given.

\subsubsection{Traffic lights}

Definitions of traffic lights were given as telemetry files in dwg format an according signal time plans given in Excel format. One may note that the Excel sheets contain "variable phases" used by the UTOPIA system to adapt the traffic lights to the current demand on the controlled roads.

\subsubsection{Traffic Demand}

Two datasets containing detector measures were supplied by the municipality of Bologna. The first one contains measures from the days 11.11.2008-13.11.2008, Tuesday to Thursday. Choosing these days is conforming to the fact that Tuesday to Thursday are usually the only "common" weekdays in means of traffic. Mondays and Fridays have different traffic shapes; on Mondays, the shape is differing due to the slightly later departure of passenger traffic and 
in some countries due to prohibitions of heavy delivery traffic on Sundays. On Fridays, the afternoon peak is often earlier, due to earlier end-of-business times.

The given measures were aggregated into intervals of half an hour. 636 detection sites were listed, where about 90 detectors (11.11.2008: 95, 12.11. 2008: 92, 13.11. 2008: 91) reported an error marked as the value " -1 ". Each of the given time line values represents the number of vehicles that passed the detection site. Because the detection is done using single induction loops, no speed information is available. Also, no distinction between different vehicle classes is given. Each detection site may cover more than one lane. The detector data is of a very good quality compare to other sites where the known number of errors in the detector data is much higher.

The second data set contains the measures for the same days, aggregated into intervals of $5 \mathrm{~min}$. The quality corresponds to the data set aggregated into $1800 \mathrm{~s}$.

The given VISSIM scenarios describe both the infrastructure and the demands within the modelled areas with a great detail and including additional information about public transport. Due to this, all information available within these inputs was imported.

Although VISSIM is a microscopic simulation just as SUMO, it follows a completely different concept of modelling the road infrastructure. The main difference is that VISSIM is not using a graph concept, consisting of nodes (intersections) and edges (roads) as SUMO does, but only of roads and connections between them. This difference makes import of VISSIM networks very complicated and the results must often be edited by hand after an initial conversion.

Because SUMO only allows importing VISSIM networks stored in German language - VISSIM uses a man-machine language for network description - the supported networks had to be translated from English into German, first. The manual validation step shown in this figure was done by comparing the network with images from Google Earth [4] and Google Maps [5], and with the supported junction telemetries. While the number of lanes was correct for all edges within the VISSIM networks, manual corrections were done on the connections between lanes over intersections.

\subsection{Demand Evaluation}

The city of Bologna has 636 detectors which are measuring the traffic flow. The measured values from three days are used for analysing the real traffic flow. Unfortunately, the detectors are measuring only the amount of vehicles which are passing the detectors within 5 Minutes there are no other values like speed or vehicle type available.

Generally, the measured traffic flow looks similar to the detector values which are shown in Figure 1 . There is only a small amount of vehicles driving during nights. In the morning hours the traffic flow is rising until there is a morning peak between 8 a.m. and 9 a.m. Afterwards, the traffic flow decreases a little bit and remains at a certain level. In the evening the traffic flow is rising to another peak. 


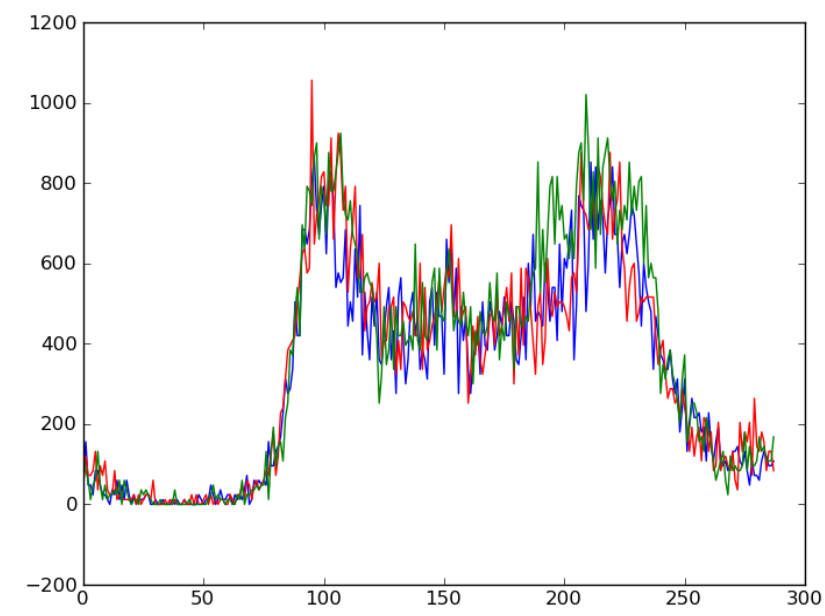

Figure 2-2: Example traffic flow of three days; blue: 11.11., red 12.11., green: 13.11.

For the validation of the simulation the real world measurements were compared to the results of the simulation. The imported networks were compared against the supplied induction loop values. Here, only the flow over the detectors were given, and were used for the evaluation. In the following, comparisons of the demands imported from VISSIM files against measures from the real world.

Table 2-4: Comparison of the simulated traffic flow compare to the measured traffic flow from the detector data. (Top to bottom: Andrea Costa, Pasubio, Joined; Left: Simulation of one hour, Right: whole simulation run).

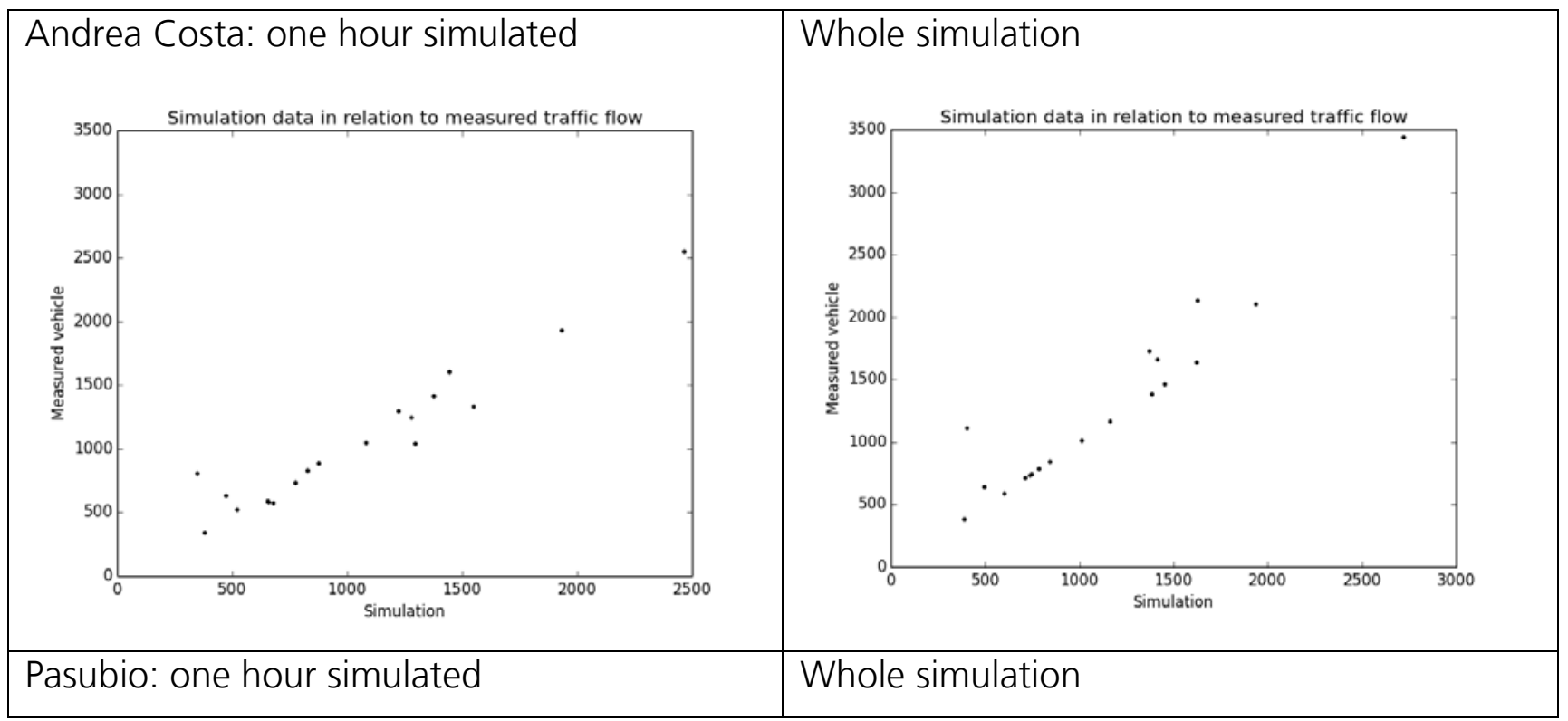




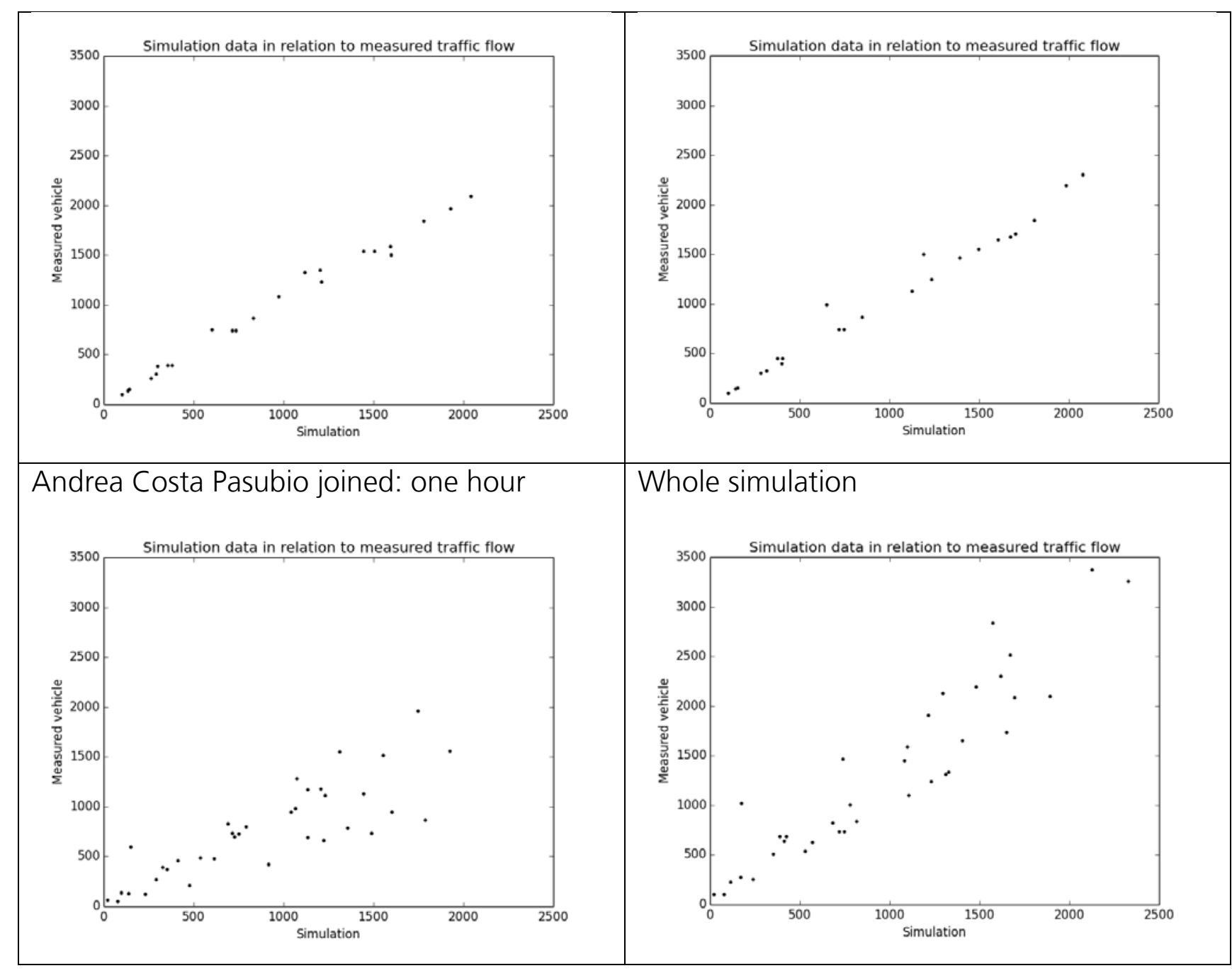

Table 2-4 shows the comparison of the average values of real measures from all detectors within the according area for the time between 8:00 and 9:00 against the ones obtained from the simulation using the demands imported from VISSIM. Two comparisons are given for each scenario: the left one shows the comparison between the real flow and the simulation results for the vehicles which passed the simulated detectors within the same time. Within the right comparison, the real world measures are put against all simulated vehicles (until the simulation ends). For both, the optimal results would be a bisectrix.

From all results the following can be seen: The overall number of vehicles which are simulated in SUMO are relatively well (right). Also the simulation results for one hour are not bad, but it can be seen that the simulation has problems to simulate the traffic demand in the joined scenario within one hour. The reason is very straight forward: with a growing areal size of the scenario, the vehicles need a longer time to populate it and cross the available induction loops. As a conclusion, it should be stated that a certain amount of simulation time is needed to fill the scenario with vehicles, before the real network state is reached. This simulation "warm-up" is a known need within traffic simulations. Normally, double of the maximum travel time through the network is used.

\subsection{User guidelines}

The Bologna scenarios are a good way to start traffic simulation based research with. The provided network, traffic demand and additional infrastructure data is broad and a lot of work was done on improve the simulation quality. For first simulations with real world data 
the scenarios can easily be used with less effort. But the use of the scenarios is also limited. For one hand side, the given traffic demand is only for one hour. Considering also a warming up and cooling down phase of the simulation, there is only approximately 30 minutes of simulation which can be used. Furthermore, the network size of the scenarios are relatively small so the route choice in the scenarios are very limeded. So rerouting algorithms should better not be simulated. Examples for traffic management strategies which can be simulated with these scenarios can be found in [6] and [7].

\subsection{Further research}

The Bologna scenarios provide traffic networks, traffic demands and representations about the traffic infrastructure to simulate a real world scenario in SUMO. But still there are open issues which should be improved. The multi-lane roundabouts produce unrealistic traffic jams. Pedestrians are currently not included into the simulation, this issue will be handled during the COLOMBO project [8].

On the one hand side, by making the scenario available to the public researcher can use these scenarios for their purposes and on the other hand side they can improve the quality of the scenarios by their corrections and enhancements.

\section{References}

[1] Krajzewicz, D., Erdmann, J., Behrisch, M., Bieker, L.: Recent Development and Applications of SUMO - Simulation of Urban MObility. International Journal On Advances in Systems and Measurements, 5 (3\&4):128-138, December 2012.

[2] Simulation of Urban MObility, www.sumo-sim.org

[3] iTETRIS Consortium (publisher): iTETRIS Deliverable 3.2 -Traffic Modelling: ITS Algorithms, April 2010.

[4] Google, Google Earth web site, http://earth.google.com.

[5] Google, Google Maps, http://maps.google.com/

[6] Bieker, Laura und Krajzewicz, Daniel (2011) Evaluation of opening Bus Lanes for private Traffic triggered via V2X Communication. First Forum on Integrated and Sustainable Transportation Systems (FISTS), 29. Jun- 1. Jul. 2011, Wien, Österreich.

[7] Bieker, Laura (2011) Emergency Vehicle Prioritization using Vehicle-To-Vehicle Communication. Young Researchers Seminar, 8.-10. Jun. 2011, Kopenhagen, Dänemark.

[8] COLOMBO consortium (publisher): COLOMBO project's Deliverable D2.1: "Policy Definition and dynamic Policy Selection Algorithms", November 2013. 